\title{
AVALIAÇÃO DE DISCIPLINA NA MODALIDADE A DISTÂNCIA POR ESTUDANTES DE GRADUAÇÃO EM ENFERMAGEM
}

Ana Luísa Petersen Cogo', Gabriela Petró Valli Czerwinski², Débora Marie da Silva Bonmann³, Jamile Araujo Durlo ${ }^{3}$, Karema da Conceição Pereira ${ }^{3}$

${ }^{1}$ Enfermeira. Doutora em Enfermagem. Professora da Universidade Federal do Rio Grande do Sul. Porto Alegre-RS-Brasil. ${ }^{2}$ Enfermeira. Mestre em Enfermagem. Universidade Federal do Rio Grande do Sul. Porto Alegre-RS-Brasil. ${ }^{3}$ Enfermeira. Escola de Enfermagem da Universidade Federal do Rio Grande do Sul. Porto Alegre-RS-Brasil.

RESUMO: O objetivo do estudo foi analisar o desenvolvimento da disciplina 'Enfermagem e Pesquisa' na modalidade a distância em Curso de Graduação em Enfermagem, caracterizando as similaridades e as diferenças em relação ao ensino presencial. Teve abordagem quantitativa do tipo exploratória-descritiva realizada com 60 estudantes da disciplina na modalidade presencial e 53 a distância de universidade pública no estado do Rio Grande do Sul. A coleta de dados ocorreu em 2012 e 2013 por questionário e dados do ambiente virtual, analisados pela estatística descritiva (nível de significância de 5\%). Os estudantes das turmas a distância apresentaram maior proporção de profissionalização, comparados com as turmas presenciais $(p=0,007)$. A comunicação por correio eletrônico foi predominante com o envio de 266 mensagens solicitando esclarecimentos sobre exercícios. Conclui-se que existem similaridade snas avaliações das turmas nas duas modalidades de ensino, sendo que a modalidade a distância é uma alternativa aos estudantes trabalhadores. DESCRITORES: Educação em enfermagem; Educação a distância; Estudantes de enfermagem; Internet; Tecnologia educacional.

\section{COURSE ASSESSMENT IN THE DISTANCE MODE BY UNDERGRADUATE STUDENTS OF NURSING}

\begin{abstract}
The study aimed to analyze the development of the 'Nursing and Research' course in the distance mode of the Undergraduate Course in Nursing, characterizing the similarities and the differences in relation to the on-site teaching. It had a quantitative approach of the exploratory descriptive type, and was undertaken with 60 students of the on-site course and 53 of the distance learning course at a public university in the state of Río Grande do Sul. Data collection took place in 2012 and 2013, through a questionnaire and data from the virtual environment, analyzed using descriptive statistics (level of significance of $5 \%$ ). The students from the distance learning groups presented a higher proportion of professionalization in comparison with the on-site groups $(p=0.007)$. Communication using email was predominant, with 266 messages sent requesting clarification regarding exercises. It is concluded that there is similarity in the groups' evaluations of the two modes of teaching, with the distance mode being an alternative for students who work. DESCRIPTORS: Education, Nursing; Education, Distance; Students, Nursing; Internet; Educational Technology.
\end{abstract}

\section{EVALUACIÓN DE ASIGNATURA EN LA MODALIDAD A DISTANCIA POR ESTUDIANTES DE GRADUACIÓN EN ENFERMERÍA}

RESUMEN: La finalidad del estudio fue analizar el desarrollo de la asignatura 'Enfermería y Investigación' en la modalidad a distancia en Curso de Graduación en Enfermería, caracterizando las semejanzas y las diferencias acerca de la enseñanza presencial. El abordaje fue cuantitativo del tipo exploratorio descriptivo realizado con 60 estudiantes de la asignatura en la modalidad presencial y 53 a distancia de universidad pública en el estado de Rio Grande do Sul. Los datos fueron recogidos en 2012 y 2013 por cuestionario y datos del ambiente virtual, analizados por la estadística descriptiva (nível de significancia de 5\%). Los estudiantes de las clases a distancia presentaron mayor proporción de profesionalización, comparados con las clases presenciales $(p=0,007)$. La comunicación por correo electrónico fue predominante con el envío de 266 mensajes solicitando aclaramientos sobre actividades. Se concluye que hay semejanza en las evaluaciones de las clases en las dos modalidades de enseñanza, siendo la modalidad a distancia una alternativa a los estudiantes trabajadores. DESCRIPTORES: Educaciónen enfermería; Educación a distancia; Estudiantes de enfermería; Internet; Tecnología educacional. 


\section{INTRODUÇÃO}

A implantação de novas metodologias educacionais requer que as mesmas sejam avaliadas quanto a sua efetividade junto aos participantes. As atividades mediadas por computador fazem parte do cotidiano das Instituições de Ensino Superior (IES) brasileiras, porém estudos revelam que é baixa a oferta de disciplinas na modalidade a distância (EAD) em Cursos de Graduação em Enfermagem ${ }^{(1)}$, além de haver um quantitativo menor de publicações sobre a EAD em comparação ao produzido em outras áreas do conhecimento ${ }^{(2)}$.

Diferentemente do que ocorria há alguns anos, hoje os jovens brasileiros utilizam os recursos de informática com maior desenvoltura e há uma progressiva democratização do acesso à internet, especialmente nos centros urbanos ${ }^{(3)}$. Associado a essas condições, deve-se considerar algumas características que vem sendo atribuídas à utilização da EAD como a facilidade de acesso à informação, a flexibilidade temporal e espacial para estudar, a interatividade com colegas e equipe executora, além da apropriação de tecnologias com vistas à melhoria contínua da prática profissional ${ }^{(1)}$. Desta forma, percebe-se na EAD um recurso de promoção para a formação e o aprimoramento dos profissionais de Enfermagem, que inicia durante o curso de graduação ao se familiarizarem com a utilização desta tecnologia.

$\mathrm{Na}$ avaliação de disciplinas na modalidade EAD, estudantes de cursos da área da saúde destacam como aspectos que colaboram com a sua aprendizagem a adequação dos conteúdos apresentados online, a interação com o professor, a dinâmica das atividades propostas, bem como a qualidade dos objetos de aprendizagem e a facilidade no uso das tecnologias ${ }^{(4-7)}$.

A presente investigação foi realizada com o intuito de descrever a avaliação realizada por estudantes de Enfermagem de disciplina na modalidade EAD no curso de graduação, analisando sua opinião sobre a mesma e a utilização do ambiente virtual. Da mesma forma, foi realizada a avaliação das turmas na modalidade presencial desta disciplina, que ocorreram concomitantemente com o EAD, a fim de identificar os aspectos similares e distintos no processo de ensino entre essas duas modalidades.
Esta avaliação é importante como subsídio para os professores de enfermagem no planejamento e no desenvolvimento de disciplinas, especialmente as que se desenvolvem na modalidade a distância. Assim, o objetivo foi analisar o desenvolvimento da disciplina 'Enfermagem e Pesquisa' na modalidade a distância em Curso de Graduação em Enfermagem, caracterizando as similaridades e as diferenças em relação ao ensino presencial.

\section{MÉTODO}

Trata-se de pesquisa na abordagem quantitativa do tipo exploratória, descritiva, retrospectiva, pois os dados foram obtidos após os estudantes terem concluído as disciplinas ${ }^{(8)}$. O estudo foi realizado em curso de Graduação em Enfermagem de universidade pública brasileira no estado do Rio Grande do Sul, que oferece a disciplina "Enfermagem e Pesquisa" com 30 horas na terceira etapa do mesmo. No primeiro semestre letivo de 2011, ocorreu a implantação de mudanças no sistema de oferecimento de turmas, havendo a possibilidade dos estudantes optarem, conforme ordenamento de matrícula, pela disciplina na modalidade EAD ou na modalidade presencial. Os conteúdos desenvolvidos, o cronograma das atividades, as professoras e as avaliações presenciais (prova e trabalhos) foram os mesmos nas duas turmas no período de realização do estudo. $\mathrm{O}$ ambiente virtual de aprendizagem utilizado para a turma EAD foi o Moodle disponibilizado pela universidade.

A população do estudo foi constituída por 156 estudantes que cursaram a disciplina nos dois semestres letivos de 2012 e no primeiro de 2013, tanto na modalidade EAD como presencial, sendo excluídos os estudantes que não completaram a disciplina por excesso de faltas, cancelamento da matrícula ao longo do semestre, afastamento por motivos de saúde ou reprovação. Desta forma, a amostra foi constituída por 113 estudantes de graduação em Enfermagem.

$\mathrm{O}$ instrumento de coleta de dados, previamente testado, continha 14 perguntas fechadas e abordava dados de caracterização dos participantes, os conhecimentos e experiências em informática/EAD e a avaliação que realizavam da disciplina (metodologia utilizada, qualidade das interações, materiais disponibilizados 
e conteúdo disponibilizado). As perguntas referentes à avaliação da disciplina tinham como alternativas 'dentro do esperado', 'acima do esperado' e 'abaixo do esperado'. No final do questionário havia espaço para serem realizados comentários ou sugestões. Os questionários autoaplicáveis foram entregues aos estudantes nos semestres seguintes ao da realização da disciplina nos anos de 2012 e 2013, com convite realizado por contato presencial ou por correio eletrônico, assegurando que não haveria influência no conceito obtido na disciplina.

O segundo momento da coleta de dados foi a análise dos registros no ambiente virtual Moodle, realizados pelos estudantes na disciplina na modalidade EAD. Foram contabilizados a quantidade e o assunto das mensagens eletrônicas enviadas à professora ao longo das 15 semanas de realização da disciplina EAD.

Os dados foram organizados com o apoio do programa estatístico Statistical Package for Social Sciences (SPSS) versão 18.0. As variáveis contínuas foram descritas por média e desvio padrão. As variáveis categóricas foram descritas por frequências absolutas e relativas. O teste t-student foi aplicado para comparar médias entre os grupos. Na comparação de proporções entre os grupos, os testes qui-quadrado de Pearson ou exato de Fisher foram aplicados. O nível de significância adotado foi de $5 \%(p \geq 0,05)$.

Este estudo observou as orientações da Resolução 196/96(9) tendo obtido aprovação do Comitê de Ética em Pesquisa da Universidade (número 20583). No cabeçalho do questionário constavam os esclarecimentos sobre o objetivo e as finalidades do estudo, seus riscos e benefícios, o anonimato e a participação voluntária, sendo considerado o aceite em participar do estudo a devolução do mesmo preenchido.

\section{RESULTADOS}

Os participantes da turma EAD $(n=53)$ e da presencial $(n=60)$ tinham idade de 23,1 $( \pm 4,6)$ e de $22,4( \pm 7,1)$ com acesso a internet nas suas residências $(98,1 \%$ e $98,3 \%)$ principalmente por banda larga (75,5\% e 73,3\%), respectivamente. A maioria dos estudantes não realizaram cursos ou disciplinas na modalidade EAD $(64,2 \%$ e $81,7 \%)$, mas já haviam usado ambientes virtuais no curso de graduação em Enfermagem (88,7\% e $88,3 \%)$, dos quais predominou o Moodle $(95,7 \%$ e $83 \%$ ). Os estudantes das turmas no ensino a distância apresentaram maior proporção de profissionalização comparativamente a presencial $(p=0,007)$, nas demais variáveis as diferenças não foram estatisticamente significativas (Tabela 1).

Quanto à familiarização com programas de computador, os estudantes foram questionados sobre o conhecimento e a habilidade para utilizá-los tendo como opções 'muito boa', 'sei um pouco', 'tenho dificuldade' e 'não utilizo'. O correio eletrônico (90,6\% e 96,7\%) e a navegação na internet $(83,0 \%$ e 88,3\%) destacaram-se com avaliações de familiarização 'muito boa'. Na alternativa 'não utilizo' apenas o ambiente virtual de aprendizagem foi pontuado (1,9\% e 3,3\%). Não houve diferença significativa entre os grupos quanto à familiarização com os programas (Tabela 2).

Os estudantes de ensino a distância tiveram melhores expectativas que estudantes de turmas presenciais quanto à interação com a professora $(p=0,008)$ e os materiais disponibilizados para estudo $(p=0,001)$. Também os estudantes da modalidade a distância afirmaram, em maior proporção, que fariam outra disciplina EAD quando comparados com os de ensino presencial $(p=0,002)$ (Tabela 3). As expectativas que se mantiveram 'dentro do esperado' nas turmas EAD e presencial foram quanto ao conteúdo da disciplina $(69,8 \% ; 80,0 \%)$, a interação com colegas $(77,4 \%, 76,7 \%)$, o trabalho em grupo $(62,3 \%, 48,3 \%)$, sem terem apresentado associação com significância ( $p=0,171, p=0,790$, $p=0,331$, respectivamente). Se avaliada as mesmas associações em cada semestre cursado pelos estudantes (2012.1, 2012.2, 2013.1), resultados semelhantes foram obtidos. A nota média para a disciplina EAD foi de $7,9( \pm 1,9)$ ao passo que para a presencial foi de $8,1( \pm 1,1)$, não sendo a diferença estatisticamente significativa $(p=0,483)$.

Quanto às sugestões apenas $12(10,6 \%)$ estudantes opinaram. Cinco estudantes $(4,4 \%)$ sugeriram que as atividades e as dúvidas fossem melhor esclarecidas, quatro $(3,5 \%)$ que deveriam ser oferecidas mais disciplinas EAD no curso, dois $(1,8 \%)$ que a professora deveria se envolver mais e um $(0,9 \%)$ que fossem realizadas aulas presenciais.

O segundo momento da coleta de dados foi a análise das navegações e das interações que 
ocorreram no ambiente virtual Moodle, durante a realização da disciplina na modalidade EAD. Nas turmas presenciais o ambiente virtual não era utilizado, desta forma os dados apresentados a seguir se referem aos acessos realizados pelos 53 estudantes das turmas na modalidade EAD. Foram disponibilizados 43 materiais de apoio à aprendizagem, entre estes apresentações, vídeos, hipertextos, jogos educativos e indicação de sites na internet, para subsidiar a realização das atividades propostas. A leitura e o estudo destes objetos de aprendizagem eram recomendados, mas não obrigatórios. A frequência na disciplina EAD ocorria pelo registro do envio da atividade semanal proposta.

O contato entre professor e estudantes ocorria online pelo correio eletrônico, sendo que 40 (75,5\%) estudantes recorreram a essa forma de comunicação totalizando 266 mensagens. Os assuntos das mensagens foram 139 (52,3\%) para esclarecimentos sobre os exercícios, 70 (26,3\%) esclarecimentos de dúvidas sobre o funcionamento da disciplina, $34(12,8 \%)$ conflitos entre os participantes dos grupos e $23(8,6 \%)$ pedidos de auxílio.

Tabela 1 - Caracterização da amostra. Porto Alegre-RS-Brasil, 2015

\begin{tabular}{|c|c|c|c|}
\hline Variáveis & $\begin{array}{c}\text { EAD } \\
(n=53 ; 46,9 \%)\end{array}$ & $\begin{array}{c}\text { Presencial } \\
(n=60 ; 53,1 \%)\end{array}$ & $\mathbf{P}$ \\
\hline Idade (anos) - média \pm DP & $23,1 \pm 4,6$ & $22,4 \pm 7,1$ & 0,404 \\
\hline Trabalha $-\mathrm{n}(\%)$ & & & 0,007 \\
\hline Sim & $17(32,1)$ & $6(10)$ & \\
\hline Não & $36(67,9)$ & $54(90)$ & \\
\hline Com internet $-\mathrm{n}(\%)$ & $52(98,1)$ & $59(98,3)$ & 1,000 \\
\hline Tipos de internet $-\mathrm{n}(\%)$ & & & 0,873 \\
\hline Banda Larga & $40(75,5)$ & $44(73,3)$ & \\
\hline Internet 3G & $8(15,1)$ & $12(20)$ & \\
\hline Outra & $4(7,5)$ & $3(5)$ & \\
\hline Não respondeu & $1(1,9)$ & $1(1,7)$ & \\
\hline Disciplina/curso EAD anterior $-\mathrm{n}(\%)$ & & & 0,083 \\
\hline Sim & $18(34)$ & $11(18,3)$ & \\
\hline Não & $34(64,1)$ & $49(81,7)$ & \\
\hline Não respondeu & $1(1,9)$ & $0(0)$ & \\
\hline Ambiente virtual de aprendizagem- $\mathrm{n}(\%)$ & & & 0,531 \\
\hline Sim & $47(88,7)$ & $53(88,3)$ & \\
\hline Não & $5(9,4)$ & $7(11,7)$ & \\
\hline Não respondeu & $1(1,9)$ & $0(0)$ & \\
\hline \multicolumn{4}{|l|}{ Tipos de ambientes virtuais $-\mathrm{n}(\%)$} \\
\hline Moodle & $45(95,7)$ & $44(83)$ & 0,087 \\
\hline Rooda & $32(68,1)$ & $28(52,8)$ & 0,177 \\
\hline Navi & $35(74,5)$ & $33(62,3)$ & 0,275 \\
\hline
\end{tabular}


Tabela 2 - Familiarização com programas. Porto Alegre-RS-Brasil, 2015

\begin{tabular}{|c|c|c|c|}
\hline Familiarização & $\begin{array}{c}\text { EAD } \\
(n=53 ; 46,9 \%)\end{array}$ & $\begin{array}{c}\text { Presencial } \\
(n=60 ; 53,1 \%)\end{array}$ & $\mathbf{P}$ \\
\hline Editor de Texto $-\mathrm{n}(\%)$ & & & 0,522 \\
\hline Dificuldades & $1(1,9)$ & $0(0)$ & \\
\hline Sei um pouco & $12(22,6)$ & $12(20)$ & \\
\hline Muito boa & $40(75,5)$ & $48(80)$ & \\
\hline Gerador de Apresentação- $\mathrm{n}(\%)$ & & & 0,382 \\
\hline Dificuldades & $1(1,9)$ & $3(5)$ & \\
\hline Sei um pouco & $29(54,7)$ & $26(43,3)$ & \\
\hline Muito boa & $23(43,4)$ & $31(51,7)$ & \\
\hline Navegação na Internet- n(\%) & & & 0,479 \\
\hline Sei um pouco & $8(15,1)$ & $7(11,7)$ & \\
\hline Muito boa & $44(83)$ & $53(88,3)$ & \\
\hline Não respondeu & $1(1,9)$ & $0(0)$ & \\
\hline Ambiente virtual de aprendizagem- $\mathrm{n}(\%)$ & & & 0,866 \\
\hline Não utiliza & $1(1,9)$ & $2(3,3)$ & \\
\hline Dificuldades & $2(3,8)$ & $1(1,7)$ & \\
\hline Sei um pouco & $29(54,7)$ & $32(53,3)$ & \\
\hline Muito boa & $21(39,6)$ & $25(41,7)$ & \\
\hline Correio eletrônico & & & 0,250 \\
\hline Sei um pouco & $5(9,4)$ & $2(3,3)$ & \\
\hline Muito boa & $48(90,6)$ & $58(96,7)$ & \\
\hline
\end{tabular}

Tabela 3 - Expectativas sobre a disciplina realizada. Porto Alegre-RS-Brasil, 2015

\begin{tabular}{|c|c|c|c|}
\hline Expectativas & $\begin{array}{c}\text { EAD } \\
(n=53 ; 46,9 \%)\end{array}$ & $\begin{array}{c}\text { Presencial } \\
(n=60 ; 53,1 \%)\end{array}$ & $\mathbf{P}$ \\
\hline Interação com professora- $\mathrm{n}(\%)$ & & & 0,008 \\
\hline Abaixo do esperado & $1(1,9)$ & $8(13,3)$ & \\
\hline Dentro do esperado & $30(56,6)$ & $42(70)$ & \\
\hline Acima do esperado & $21(39,6)$ & $10(16,7)$ & \\
\hline Não respondeu & $1(1,9)$ & $0(0)$ & \\
\hline Materiais disponibilizados- $\mathrm{n}(\%)$ & & & 0,001 \\
\hline Abaixo do esperado & $1(1,9)$ & $8(13,4)$ & \\
\hline Dentro do esperado & $38(71,7)$ & $44(73,3)$ & \\
\hline Acima do esperado & $14(26,4)$ & $3(5)$ & \\
\hline Não respondeu & $0(0)$ & $5(8,3)$ & \\
\hline Faria (outra) disciplina EAD - $\mathrm{n}(\%)$ & & & 0,002 \\
\hline Sim & $39(73,6)$ & $26(43,3)$ & \\
\hline Não & $14(26,4)$ & $29(48,4)$ & \\
\hline Não respondeu & $0(0)$ & $5(8,3)$ & \\
\hline
\end{tabular}




\section{DISCUSSÃO}

A caracterização dos participantes desta investigação no que se refere a faixa etária, a dedicação exclusiva dos mesmos ao estudo, não estarem inseridos no mercado de trabalho, além de terem um bom conhecimento de informática coincide com achados de outro estudo ${ }^{(10)}$. A totalidade dos participantes referiram ter acesso à internet nas suas residências, confirmando dados divulgados pelo Comitê Gestor da Internet no Brasil, apontando importantes mudanças de distribuição e acesso dos usuários que estão ocorrendo no país, aspectos esses que vem a favorecer as propostas de $\mathrm{EAD}^{(3)}$. Dados do censo sobre uso das tecnologias da informação e da comunicação (TIC) de 2012 no Brasil indicaram que mesmo com diferenças regionais, na grande maioria do país cresceu a posse de computadores e de internet nas residências em relação aos anos anteriores $^{(3)}$.

Assim, pode-se constatar que não foi por falta de acesso aos recursos tecnológicos ou falta de conhecimento no uso da informática que os participantes da pesquisa não realizaram cursos na modalidade EAD anteriormente. Existem ainda ideias pré-concebidas que não valorizam as propostas na modalidade EAD, considerandoas de baixa qualidade ou com ausência de interação, o que não condiz com a realidade. Desta forma, a ampliação da oferta de disciplinas EAD oportuniza que os estudantes vivenciem esta modalidade que para eles é uma novidade, e possam fazer opções mais adequadas ao seu estilo de aprendizagem.

A maior concentração de estudantes trabalhadores nas turmas EAD da disciplina em estudo, indica que a organização do tempo e do local de estudo influenciou a opção de matrícula de alguns estudantes. A facilidade em estudar fora das dependências da universidade e em horários mais flexíveis são vantagens já conhecidas desta modalidade colaborando para que trabalhadores possam se qualificar no Ensino Superior. Em outros países, os chamados 'estudantes não tradicionais', fizeram com que as universidades se adaptassem a esta nova demanda oferecendo como opções disciplinas na modalidade EAD no Curso de Enfermagem ${ }^{(11)}$.

A avaliação da disciplina EAD realizada pelos estudantes obteve escores satisfatórios em quase todos os quesitos, sendo que os mesmos indicaram que fariam outra disciplina nesta modalidade, indicativo de que a mesma correspondeu as suas expectativas. Mas, alguns aspectos merecem destaque, mesmo não tendo significância estatística, entre esses estavam o trabalho em grupo e a interação com os colegas.

A integração entre os colegas, para a realização de atividades coletivas, é um dos desafios do ensino mediado por computador, o qual deve oportunizar a construção de uma comunidade de aprendizagem com espaços de trocas entre todos os participantes ${ }^{(12)}$. Os conflitos nos momentos de trabalho em grupo são frequentes na modalidade presencial, mas devido às particularidades da EAD podem se intensificar, pois não há a presença física e as interações são mediadas por meio do computador. Assim, caberá ao professor identificar a ocorrência dos conflitos e gerenciar os mesmos para promovera aprendizagem.

O fato dos estudantes da disciplina EAD manifestarem uma avaliação acima do que esperavam quanto à interação com a professora e na qualidade do material disponibilizado, remete ao fato de que as diversas formas de comunicação na EAD são aspectos principais para sua efetividade, devendo ser objetiva e clara para que os participantes das atividades consigam obter respostas aos seus questionamentos de forma autônoma ${ }^{(12)}$.

O material desenvolvido para ser disponibilizado aos estudantes EAD procurou contemplar a interatividade, suscitando em questionamentos que foram esclarecidos com o professor ou com o tutor promovendo uma aprendizagem ativa. No ensino tradicional existe uma dependência do professor e o foco na tarefa em detrimento de aprender novos conhecimentos. A implantação de metodologias ativas com o apoio de materiais digitais rompe com o ensino expositivo-receptivo das aulas presenciais, propõe um estudante mais autônomo e familiarizado com o uso destas tecnologias ${ }^{(13-14)}$. Assim, considera-se essa uma fase inicial de transição, na qual progressivamente os estudantes assumirão seu protagonismo no processo de aprendizagem. 


\section{CONCLUSÃO}

A análise do desenvolvimento da disciplina no Curso de Graduação em Enfermagem na modalidade a distância em comparação com a turma presencial possibilitou identificar similaridades entre os estudantes, no que se refere a faixa etária, acesso à internet e conhecimentos de informática. Nas turmas EAD havia mais estudantes trabalhadores, sendo opção para os que necessitaram conciliar o estudo e o trabalho.

A avaliação dos estudantes nas duas modalidades apresentaram semelhanças, indicando que a EAD, superou expectativas de interação com o professor e de disponibilização de materiais que apoiaram a aprendizagem. A organização do tempo de estudo oferecida pela disciplina incentiva a autonomia no processo de aprendizagem, trazendo ao estudante maior liberdade de tempo e de espaço para a busca pelo conhecimento, mas estes necessitam conquistar seu protagonismo no processo de aprender.

Recomenda-se que estudos de acompanhamento de disciplinas na modalidade EAD nos cursos de Graduação em Enfermagem possam ser realizados, com o objetivo de avaliar as diferentes estratégias de ensino e as ferramentas de apoio utilizadas. Da mesma forma, acreditase que possam ser aprimoradas a comunicação entre professor e estudantes no esclarecimento de dúvidas, as estratégias que possibilitem trabalho em grupo cooperativo e a promoção da melhor interação entre os participantes.

\section{REFERÊNCIAS}

1. Rojo PT, Vieira SS, Zem-Mascarenhas SH, Sandor ER, Vieira CRSP. Panorama da educação à distância em enfermagem no Brasil. Rev. esc. enferm. USP. 2011;45(6):1476-80.

2. Camacho ACLF. Análise das publicações nacionais sobre educação à distância na enfermagem. Rev. bras. enferm. 2009;62(4):588-93.

3. Comitê Gestor da Internet no Brasil (CGIT). TIC Domicílios e Empresas 2012: pesquisa sobre o uso das tecnologias da informação e comunicação no Brasil. São Paulo: CGIT; [Internet] 2013. [acesso em 15 Jan 2014]. Disponível: http://www.cetic.br/ publicacoes/2012/tic-educacao-2012.pdf satisfaction with e- Learning experience in King Khalid University, Saudi Arabia. Int Jof Learn\&Develop. 2012;2(2):201-15.

5. Perumalla C, Mak J, Kee N, Matthews S. Integrating web applications to provide an effective distance online learning environment for students. Procedia Comput Sci. 2011;3:770-84.

6. Koch J, Andrew S, Salamonson Y, Everett B, Davidson PM. Nursing students' perception of a web-based intervention to support learning. Nurse edc. today. 2010;30(6):584-90.

7. Frota NM, Barros LM, Araújo TM, Caldini LN, Nascimento JC, Caetano JA. Construção de uma tecnologia educacional para o ensino de enfermagem sobre punção venosa periférica. Rev. gauch. enferm. 2013;34(2):29-36.

8. Tobar F, Yalour MR.Como fazer teses de saúde pública. Rio de Janeiro: Editora Fiocruz, 2003.

9. Ministério da Saúde (BR). Conselho Nacional de Saúde. Resolução n. 196, de 10 de outubro de 1996. Diretrizes e normas regulamentadoras de pesquisas envolvendo seres humanos. Brasília; 1996.

10. Alves ED, Ribeiro LSN, Guimarães DCSM, Costa CMA, Peixoto HM, Martins EF, et al. Moodle-fólio para o ensino em saúde e enfermagem: avaliação do processo educacional. Rev. Eletr. Enf. [Internet] 2012;14(3) [acesso em 28 nov 2014]. Disponível: http:// www.fen.ufg.br/revista/v14/n3/pdf/v14n3a03.pdf

11. Dearnley C, Dunn G, Watson S. An exploration of on-line access by non-traditional students in higher education: a case study. Nurse edc. today. 2006;26:409-15.

12. Boling EC, Hough M, Krinsky $H$, Saleem H, Stevens $M$. Cutting the distance in distance education: perspectives on what promotes positive, online learning experiences. Internet and Higher Educ. 2012;15:118-26.

13. Carmenado IR, Díaz-Puente JM, Gajardo FG. Behavior competence development through e-learning: experience at the undergraduate level in the context of aula a distancia abierta (ADA) Madrid, Spain. Procedia Soc and Behav Sci. 2011;15:111-19.

14. Frota N, Barros LM, Costa AFA, Santos ZMSA, Caetano JA. Hipermídia educacional sobre punção venosa periférica: perspectiva de acadêmicos de enfermagem. Cogitare Enferm. 2014;19(4):717-25.

4. Ali WGM. Factors affecting nursing student's 